\title{
A MATHEMATICAL MODEL OF ACOUSTIC THERMAL EXCITATION FOR PULSE TUBE ENGINE
}

\author{
E.A. Zinovyev, A.I. Dovgyallo, S.O. Nekrasova*
}

\begin{abstract}
Thermal Engineering and Heat Engines, Aircraft Engines and Power Plants, Samara State Aerospace University, 34, Moskovskoe shosse, 443086 Samara, Russia. Corresponding author's e-mail: yhoji@yandex.ru
\end{abstract}

Keywords: pulse tube engine, nonlinear processes, transient mode, standing-wave.

\section{Introduction}

The most of works applied for the pulse tube converters contain the workflow description implemented through the use of mathematical models on stationary modes. However, the study of the thermoacoustic systems unsteady behavior in the start, stop, acoustic load changes modes is in the particular interest. Yuan et.al. [1] offered a simplified quasi-onedimensional model of a thermoacoustic engine that described the amplification of acoustic oscillations and their subsequent "saturation" at the launch phase. For the study of the standing wave thermoacoustic engines functioning in the start mode Karpov et.al. [2] applied a weakly non-linear theory of the thermoacoustic instability in a channel with temperature gradient. The motivation for this work was provided by the research results of the lumped thermal capacity model presented by Matveev [3]. The aim of the present study is to develop a mathematical thermal excitation model of acoustic oscillations in pulse the tube engine (PTE) (Figure1) in more general formulation of the mathematical description. A half-length cylindrical resonator length is $240 \mathrm{~mm}$, resonator radius $-10 \mathrm{~mm}$, regenerator length $-60 \mathrm{~mm}$, porosity -0.89 , piston mass $-0.02 \mathrm{~kg}$.

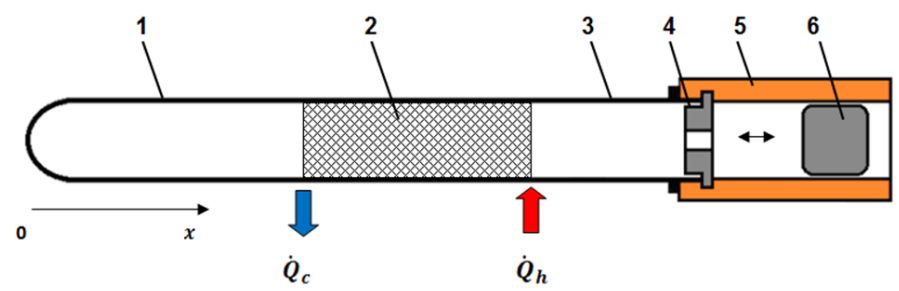

Figure 1: Scheme of the pulse tube engine

1 -resonator; 2 -regenerator; 3 -pulse tube; 4 - jet nozzle; 5 -cylinder; 6 -piston.

The model assumes the assignment of any porosity (for stack or regenerator), takes the piston weight and the friction in the cylinder and piston unit into account, and determines the operating frequency (in contrast to [3], where the fundamental acoustic mode was considered). The numerical method is based on the relation equations between the pressure and volume velocity variables at the ends of each element of PTE which is recorded through the appropriate transformation matrix. A solution demonstrates that the PTE operation frequency is the complex value, and it depends on the piston mass and the dynamic friction due to its movement in the cylinder. The real part of frequency variable determines the actual operating frequency of the engine, and the imaginary determines the damping factor of the oscillations. On the basis of the determined frequency thermoacoustically induced heat transport and generation of acoustic power equations were solved for channel with temperature gradient on its ends. This model was applied to a small-scale scheme of pulse 
tube engine (Figure1) operating at atmospheric air. Unlike the work [3] this standing wave configuration is a fully closed system. The results of numerical simulation demonstrate the features of the initialization process of PTE and show that high-amplitude acoustic oscillations can occur in the initial moment of time (Figure2) as [3] presented. The fact that generated acoustic power more than power on the steady mode in a factor of $3 \ldots 4$ doesn't mean the possibility of its it's further continuous utilizing due to its existence only in transient mode which lasts only for a 30-40 sec.

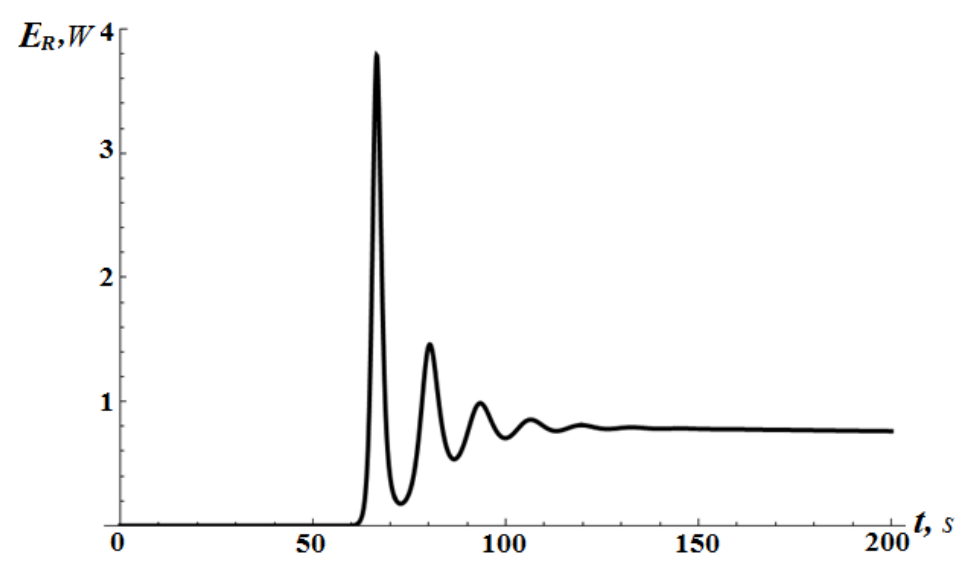

Figure 2: Acoustic power generated in the regenerator.

The experiments were carried out on small-scale scheme of the pulse tube engine. The data registration of the pressure changing as a function of the piston length displacement on the oscilloscope was implemented. The results shows that the value of acoustic power $W_{a c}$ is in the range of $0.7 . .1 .05 \mathrm{~W}$ for the defined frequency range $f=13 \ldots 18 \mathrm{~Hz}$ and pressure amplitudes $11 \ldots 12 \mathrm{kPa}$. These experimental data are satisfactorily correlated with the numerical modeling results. The mathematical model can be straightforwardly applied for the thermoacoustic devices with variable temperatures of thermal reservoirs and variable transduction loads which are expected to occur in practical implementations of portable thermoacoustic engines.

\section{References}

[1] Yuan H., Karpov S., Prosperetti A. "A simplified model for linear and nonlinear processes in thermoacoustic prime movers. Part II. Nonlinear oscillations", J. Acoust. Soc. Am., 102 (6), (1997), 3497-3506.

[2] Karpov S., Prosperetti A. "Nonlinear saturation of the thermoacoustic instability", J. Acoust. Soc. Am., 107 (6), (2000), 3130-3147.

[3] Matveev K.I. "Unsteady model for standing-wave thermoacoustic engines", J. Non-Equilib. Thermodyn., 35(2), p. 85-96. 